\title{
Power System Automation with CAS Using Wireless Sensor Network (ZIGBEE)
}

\author{
Balamurugan . $\mathbf{M}^{1}$, Parthiban . $\mathbf{T}^{2}$ \\ ${ }^{1,2}$ Assistant Professor, Department of EEE, Excel College of Engineering and Technology, Namakkal -637303
}

\begin{abstract}
Electricity losses in India during transmission and distribution are extremely high and vary between 30 to $45 \%$. Actually there is less than $10 \%$ of electrical power loss in transmission loss but power theft is mistakenly taken as transmission loss. Here our proposed system is restricting the power theft and unwanted losses. Here we used Wireless network based architecture for monitoring and optimizing the electric transmission and distribution system. The system consists of distributed power measurement node, consumer power measurement node and monitoring and control unit. Here we use Zigbee for communicating data between sensor nodes, Microcontroller for collecting data from energy meter and Visual Basic(VB)based Graphical User Interface (GUI) for monitoring power theft and other associated problems. We added one more innovative and new concept to distribute the available electrical energy to all consumer during power demand through Conditional access system (CAS) Mode.
\end{abstract}

Keywords: ZIGBEE, CAS, GUI, VB

\section{Introduction}

The world today's facing the most critical Problem of not getting the regular power. In many countries, people had not getting at least the primary needs of lights, fans, TV etc. In nearly every country, researchers expect existing energy production capabilities will fail to meet future demand without new sources of energy, including new power plant construction. However, these supply side solutions ignore another attractive alternate which is to slow down or decrease energy consumption through the use of technology to dramatically increase energy efficiency.

To manage the available power more often the power is cut for particular area, and that area goes in dark i.e. not even a single bulb can work. Instead, we can use available power in such a way that only low power devices like Tubes, Fans, Desktops and TV, Which are primary needs, should be allowed and high power devices like heater, pump-set, A.C. etc should not be allowed for that particular period. To achieve this, system can be created which will differentiate between high power and low power devices at every node and allow only low power devices to be ON.

To achieve this system we create a wireless sensor network having number of nodes which communicate with each other in full duplex mode. The communication will consist of data transfer, controlling node operation.

We are using zigbee protocol for the wireless communication. The main advantage of using ZigBee protocol is that the nodes require very less amount of power so it can be operated from battery. And in this way we have managing the available power by using wireless sensor network working on zigbee protocol. Each node is measuring the power, which is being consumed by the appliances.

The appliance is controlled by the end device i.e. node. An overall operation of the system is controlled by the control device. The Main purpose of the project is that the wireless sensor network will differentiate and control the devices in the network on the basis of power consumed by appliances to make the efficient use of power. The basic parts of the project include a Control Unit, End Device Unit having ZigBee interface

\subsection{Objectives}

- To prevent total power shutdown.

- To detect transmission loss, Insulation failures \& power theft occurring in the transmission line.

- To provide communication between user and service provider

- To calculate power readings \& power tariff

- Electricity losses in India during transmission and distribution are extremely high and vary between 30 to $45 \%$.

- Actually there is less than $10 \%$ of electrical power loss in transmission loss but power theft is mistakenly taken as transmission loss.

- Here we use Zigbee for communicating data between sensor nodes.

- Here we add one more innovative and new concept for distribute available electrical energy to all consumers during power demand through Conditional access system (CAS) Mode.

\section{ZIGBEE TECHNOLOGY AND ITS APPLICATION IN WIRELESS HOME AUTOMATION SYSTEMS: A SURVEY}

Wireless home automation systems have drawn considerable attentions of the researchers for more than a decade. The major technologies used to implement these systems include Z-Wave, Insteon, Wavenis, Bluetooth, WiFi, and ZigBee. Among these technologies the ZigBee based systems have become very popular because of its low cost and low power consumption. In this paper ZigBee based wireless home automation systems have been addressed. There are two main parts of this paper. In the first part a brief introduction of the ZigBee technology has been presented and in the second part a survey work on the ZigBee based wireless home automation system has been presented. The performances of the ZigBee based systems have also been 


\section{International Journal of Science and Research (IJSR) \\ ISSN (Online): 2319-7064 \\ Index Copernicus Value (2013): 6.14 | Impact Factor (2014): 5.611}

compared with those of other competing technologies based systems.

\section{Output Module}

\subsection{Distribution Side}

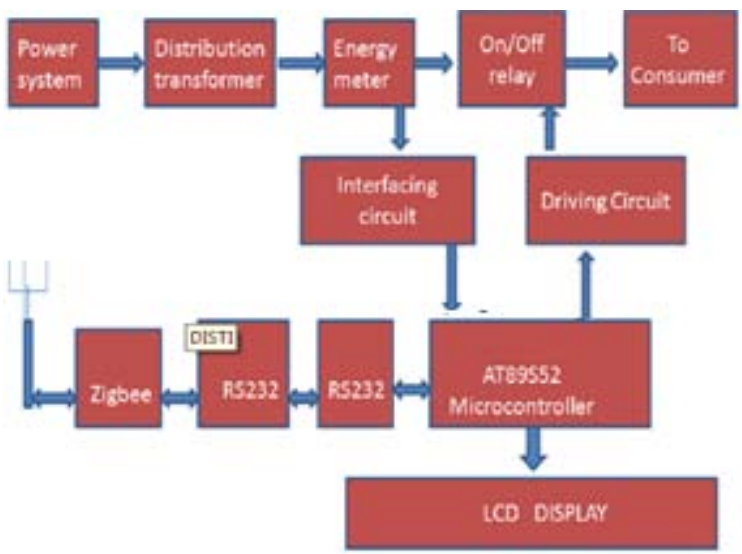

Figure 2.1: Block Diagram of distribution side

In this section, as the name suggests, these system will be installed at the distribution transformer side. Here we are fixing one energy meter in secondary of distribution transformer and amount of delivered electrical power is taken from the energy meter through sensing unit and one relay for tripping electrical energy to associated consumer area during power theft and associated problem. After that microcontroller is used for transmitting and receiving signal data from monitoring and control unit through Zigbee based wireless network.

The bulk transfer of electrical energy, from generating power plants to electrical substations located near demand centers. This is distinct from the local wiring between highvoltage substations and customers, which is typically referred to as electric power distribution. Transmission lines, when interconnected with each other, become transmission networks. The combined transmission and distribution network is known as the "power grid" in North America, or just "the grid". In the United Kingdom, the network is known as the "National Grid".

\subsection{Monitoring Side}

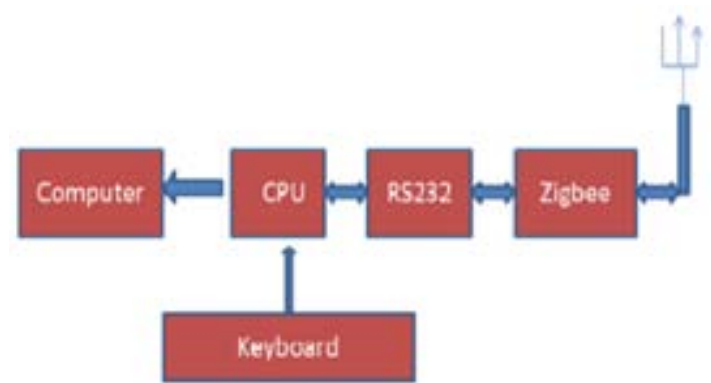

Figure 2.2: block diagram of monitoring side

In this section, as the name suggests, these system will be installed at the nearby consumer area. We need to install the system to keep a track on the amount of power transmitted from distribution side and the amount of power received in consumer side. For that, there is a wireless node, which is actually receiving the data from other wireless nodes. The Data are processed by using application program which is developed by Visual Basics. The developed application program continuously checks the amount of power delivered from distribution side and amount of power received in consumer side. If there is a major difference in between these values, then a alarm is set in the monitor, where the officials get the information about power theft and associated problems in transmission line and sending information to the tripping circuit to isolate the particular area.

\subsection{Consumer Side}

In this section, as the name suggests, these system will be installed at the consumer side. We need to install our sensing unit to existing energy meter. This system is to get the correct amount of power delivered to consumer.

Now we need to send this information to monitoring station, for that we need to use the wireless network. But there is a problem, that we can't connect the wireless network directly to the power sensing unit.

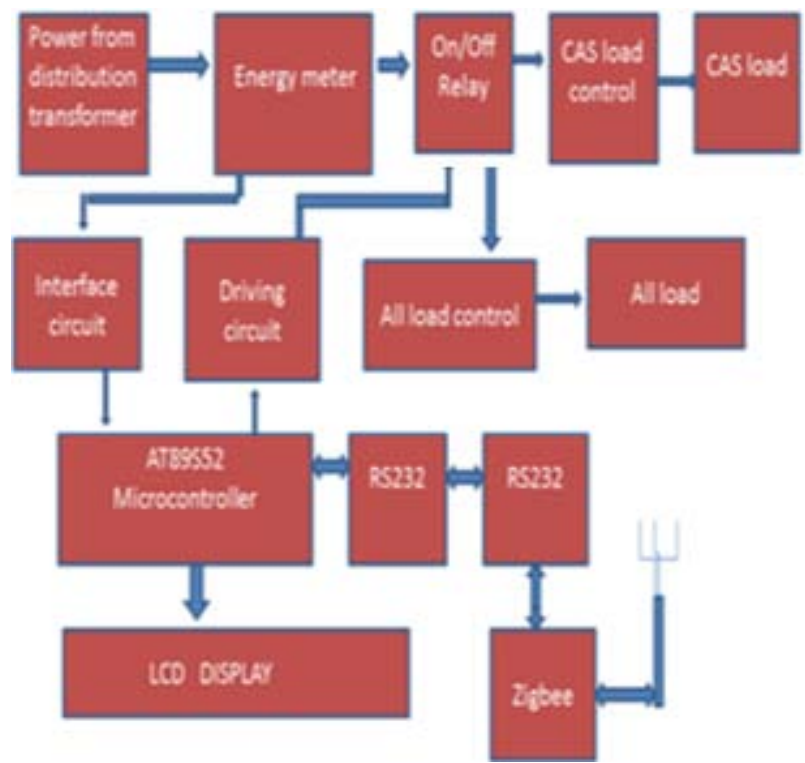

Figure2.3: block diagram of consumer side

We have to connect it through a heck of circuits, to avoid it, we can use microcontroller. So, we are actually using microcontroller to send the information from sensing unit to monitoring station through wireless network.

\section{Step Down Transformer}

When AC is applied to the primary winding of the transformer, it can either be stepped down or stepped up depending on the value of DC needed. In our circuit the transformer of $230 \mathrm{v} / 15 \mathrm{v}$ is used to perform the step down operation where a $230 \mathrm{~V}$ AC appears as $5 \mathrm{~V}$ AC across the secondary winding. The current rating of the transformer used in our project is 1A. Apart from stepping down AC voltages, it provides isolation between the power source and power supply modules. 


\section{International Journal of Science and Research (IJSR) \\ ISSN (Online): 2319-7064}

Index Copernicus Value (2013): 6.14 | Impact Factor (2014): 5.611

\subsection{Rectifier Unit}

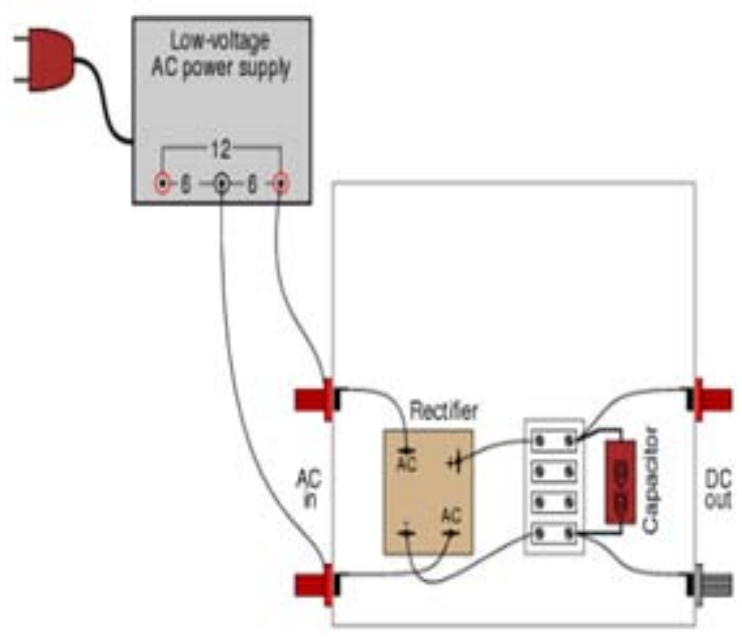

Figure 3.2: Power supply block

In the power supply unit, rectification is normally achieved using a solid state diode. Diode has the property that will let the electron flow easily in one direction at proper biasing condition. As AC is applied to the diode, electrons only flow in forward bias (anode is positive and cathode is negative).In reverse biasing (anode is negative and cathode is positive) the polarity of voltage will not permit electron flow.

A commonly used circuit for full wave rectification for large amounts of DC power is achieved by bridge rectifier circuit, bridge rectifier is constructed using four diodes (IN4007x4) is used to achieve full wave rectification.

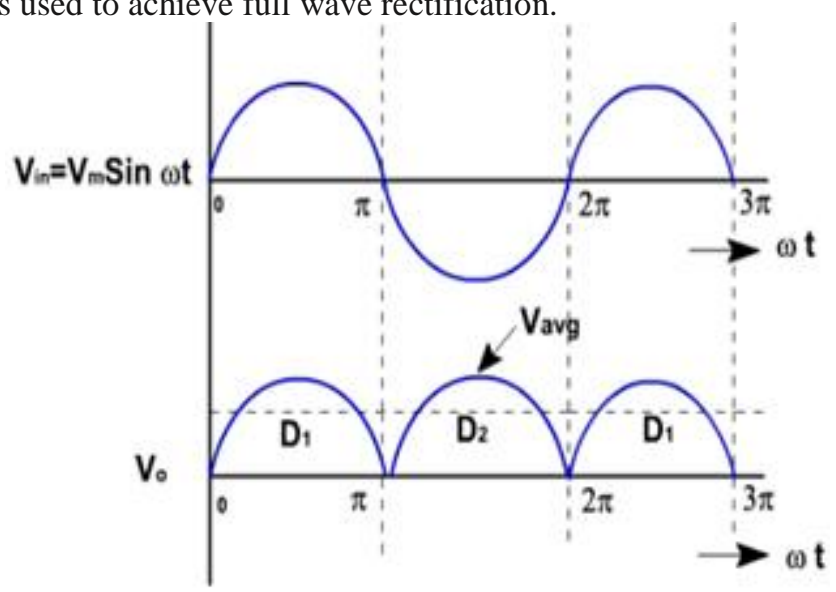

Figure 3.3: waveforms of bridge rectifier

Two diodes will conduct during the first half cycle and the other two diodes will conduct during the next half cycle. The DC voltage appearing across the output terminals of the bridge rectifier will be somewhat less than $90 \%$ of the applied rms value.

With a regulator connected to the DC output, the voltage can be maintained within a close tolerant region for the desired output.. They are exceptionally easy to use and require only two external resistors to set the output voltage. Further, both line and load regulations are better than the standard fixed regulators. Also, the L7800 series is packaged in standard transistor packages which are easily mounted and handled. In addition to higher performance than fixed regulators, the
L7800 series offers full overload protection available only in IC's (Transformer + Rectifier + Smoothing + Regulator).

\section{Description}

The power supply unit consists of transformer, rectifier circuits, filter and regulator. Step down transformer is used to step down the voltage. The ratings of transformer are $230 \mathrm{vac} / 12 \mathrm{v}, 50 \mathrm{hz}$. the lowered ac voltage is converted into dc by using rectifier circuits. bridge rectifier is used for its advantages over other types. this voltage obtained from the rectifier is regulated and regulated dc voltage is given to the other units.

\section{Brief Description of CAS}

All Conditional Access systems for satellite, terrestrial and cable broadcast use the Common Scrambling Algorithm (CSA) to perform the actual encryption and decryption of the TV signals. In the CSA specification this is known as scrambling and descrambling. Some vendors focusing solely on Conditional Access systems for IP-TV have chosen other scrambling algorithms but CSA remains the de facto standard.

The clear signal is fed into the scrambler which encrypts using the Control Word. The descrambler uses the same Control Word to recreate the original clear signal. The clear (not encrypted) signal is fed into a scrambler which encrypts the signal using a random encryption key called the Control Word (CW) according to the CSA algorithm.

In a normal DVB system, the scrambling takes place in a multiplexer or an IP Streamer, while the descrambling takes place in a set-top box (STB).

The Control Word used for scrambling is chosen randomly. This is normally done by the device performing the scrambling.

The Simulcrypt specification as well as the MPEG and DVB specifications regulate how the information is sent in the signals and how the information needed by the CA system is to be transmitted.

For security reasons the Control Word used for scrambling is changed regularly, usually every 10 seconds. The transport stream header contains two scrambling control bits which indicate which Control Word are to be used for descrambling, normally denoted Odd and Even. This allows the Control Words to be changed without causing any descrambling problems

\section{RS-232 Drivers}

The typical driver output voltage swing is $\pm 8 \mathrm{~V}$ when loaded with a nominal $5 \mathrm{k} \Omega$ RS-232 receiver and $\mathrm{VCC}=+5 \mathrm{~V}$. Output swing is guaranteed to meet the EIA/TIA- 232E and V.28 specification, which calls for $\pm 5 \mathrm{~V}$ mini- mum driver output levels under worst-case conditions. These include a minimum $3 \mathrm{k} \Omega$ load, $\mathrm{VCC}=+4.5 \mathrm{~V}$, and maximum operating temperature. Unloaded driver out- put voltage ranges from $(\mathrm{V}+-1.3 \mathrm{~V})$ to $(\mathrm{V}-+0.5 \mathrm{~V})$. Input thresholds are both TTL 


\section{International Journal of Science and Research (IJSR) \\ ISSN (Online): 2319-7064 \\ Index Copernicus Value (2013): 6.14 | Impact Factor (2014): 5.611}

and CMOS compatible. The inputs of unused drivers can be left unconnected since $400 \mathrm{k} \Omega$ input pullup resistors to VCC are built in (except for the MAX220). The pullup resistors force the outputs of unused drivers low because all drivers invert.

The internal input pull up resistors typically source $12 \mu \mathrm{A}$, except in shutdown mode where the pullups are dis- abled. Driver outputs turn off and enter a high-imped- ance statewhere leakage current is typically microamperes (maximum $25 \mu \mathrm{A}$ ) - when in shutdown mode, in three-state mode, or when device power is removed. Outputs can be driven to $\pm 15 \mathrm{~V}$.

\section{ZIGBEE}

ZigBee The ZigBee standard is built on top of the IEEE 802.15.4 standard. The IEEE 802.15.4 standard defines the physical and MAC (Medium Access Control) layers for lowrate wireless personal area networks . The physical layer supports three frequency bands with different gross data rates:2,450 MHz (250 kbs-1), $915 \mathrm{MHz}$ (40 kbs-1) and 868 MHz (20 kbs-1) It also supports functionalities for channel selection, link quality estimation, energy measurement and clear channel assessment. ZigBee standardizes both the network and the application layer.

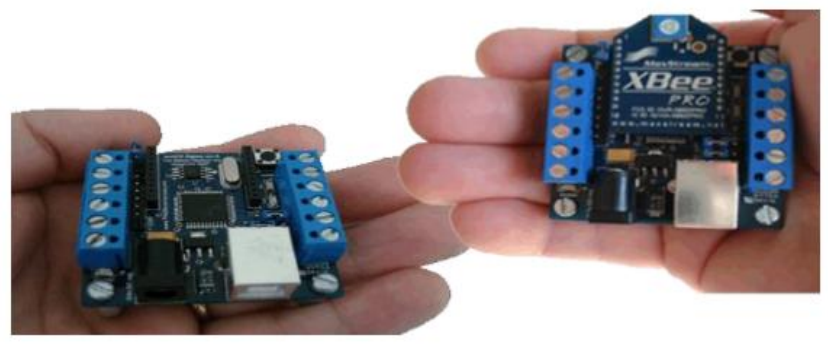

Figure 5.2 zigbee model

The network layer is in charge of organizing and providing routing over a multi-hop network, specifying different network topologies: star, tree, peer-to-peer andmesh. The application layer provides a framework for distributed application development and communication. Aside from the agriculture and food industry, it is widely used in home building control, automation, security, consumer electronics, personal computer peripherals, medical monitoring and toys. These applications require a technology that offers long battery life, reliability, automatic or semiautomatic installation, the ability to easily add or remove network nodes, signals that can pass through walls and ceilings and a low system cost.

\section{Conclusion}

Our innovation could help the energy distribution companies, reduce costs, increase profits, improve billing accuracy, efficiency, contribute energy sustainability, reduce man power for billing and also reduce unwanted losses in transmission and distribution system. By using this innovation, electrical power distribution companies can totally eliminate the power theft in distribution system. Compare with existing system, our system is having following features

It totally stop bogus seals, tampering of seals \& meter tampering.
Here zigbee network used, which is easily available, quick accessibility, having high range and easily extendable.

It will indirectly enhance the national economy. It totally restrict the power theft.

Our innovation could help the energy distribution companies to:

- Improve billing accuracy Efficiency, contribute to energy sustainability Reduce man power for billing

- Unwanted losses in transmission and distribution system. By using this innovation electrical power distribution companies totally eliminate the power theft in distribution system.

\section{References}

[1] Qixun Yang, Board Chairman, Beijing Sifang Automation Co. Ltd., China and .Bi Tianshu, Professor, North China Electric Power University, China. (2001-0624). "WAMS Implementation in China and the Challenges for Bulk Power System Protection" (PDF). Panel Session: Developments in Power Generation and Transmission - Infrastructures in China, IEEE 2007 General Meeting, Tampa, FL, USA, 24-28 June 2007 Electric Power, ABB Power T\&D Company, and Tennessee Valley Authority (Institute of Electrical and Electronics Engineers). Retrieved 2008-12-01.

[2] [Jones01] Christine E. Jones, Krishna M. Sivalingam, Prathima Agrawal, Jyh Cheng Chen. A Survey of Energy Efficient Network Protocols for Wireless Networks. Wireless Networks. Volume 7, Issue 4 (August 2001). Pg. 343-358. ISSN: 1022-0038 .

[3] Yilu Liu, Lamine Mili, Jaime De La Ree, Reynaldo Francisco Nuqui, Reynaldo Francisco Nuqui (2001-0712). "State Estimation and Voltage Security Monitoring Using Synchronized Phasor Measurement" (PDF). Research paper from work sponsored by American Electric Power, ABB Power T\&D Company, and Tennessee Valley Authority (Virginia Polytechnic Institute and State University)... Retrieved 2008-12-01. Abstract lay summary. "'Simulations and field experiences suggest that PMUs can revolutionize the way power systems are monitored and controlled"'.

[4] Olaf Stenull; Hans-Karl Janssen (2001). "Nonlinear random resistor diode networks and fractal dimensions of directed percolation clusters". Phys. Rev. E 6435 (2001) 64. 